\title{
Three-dimensional numerical simulation of debris flow using the finite volume method
}

\author{
Ruben A. Figueroa-León, Ing. ${ }^{1}$, and Serapio A. Quillos-Ruiz, Dr. ${ }^{1}$ \\ ${ }^{1}$ Universidad Nacional del Santa, Perú,rfigueroa2709@gmail.com, s_quillos@hotmail.com
}

\begin{abstract}
OpenFOAM free software uses computational fluid dynamics through finite volume method; in adition, works the debris flow model with 98\% precision in mesh refinement tests, applying the LES turbulence model: oneEqEddy. Likewise, the advance of the flow was predicted in a more consistent way, getting a behavior closer to the real one of the fluid under study comparing with other methods. In experimental model, average speeds between $2.5784 \mathrm{~m} / \mathrm{s}$ and $2.1076 \mathrm{~m} / \mathrm{s}$ were obtained, and a range $R 2$ determination coefficients of 0.997 to 0.9903; and in the numerical model an average speed of $2.0572 \mathrm{~m} / \mathrm{s}$ to $R 2$ of 0.974 were obtained, being the statistically similar slopes, providing the numerical model a good level of reliability to be used to simulate different behaviors of debris movement in ravines on a natural scale with accurate predictions. The threedimensional model of debris flows reproduced the wavefront velocities with $85 \%$ precision, coinciding in shape and time in each control section of the physical model; Similarly, turnbuckles have gotten $80 \%$ accuracy, reach, and displacement of the debris flow within the ejection cone. The results of the experimental model and of the variable simulations stablish possible to model the debris flows in complex terrains with good precision.
\end{abstract}

Keywords - Debris flow, OpenFOAM software, numerical simulation, experimental ravines.

Digital Object Identifier (DOI):

http://dx.doi.org/10.18687/LACCEI2020.1.1.573

ISBN: 978-958-52071-4-1 ISSN: 2414-6390

$1^{\text {th }}$ LACCEI International Multi-Conference for Engineering, Education, and Technology: "Engineering, Integration, and Alliances for a Sustainable Development" "Hemispheric Cooperation for Competitiveness and Prosperity on a Knowledge-Based Economy", July 27-31, 2020, Virtual Edition. 


\title{
Simulación numérica tridimensional de flujos de escombros utilizando el método de volúmenes finitos
}

\section{Three-dimensional numerical simulation of debris flow using the finite volume method}

\author{
Ruben A. Figueroa-León, Ing. ${ }^{1}$, and Serapio A. Quillos-Ruiz, Dr. ${ }^{1}$ \\ ${ }^{1}$ Universidad Nacional del Santa, Perú, rfigueroa2709@gmail.com, s_quillos@ hotmail.com
}

\begin{abstract}
Resumen - El software libre OpenFOAM utiliza la dinámica de fluidos computacional mediante el método de volúmenes finito y trabaja el modelo de flujos de escombros con una precisión del $98 \%$ en las pruebas de refinamiento de malla, con la aplicación del modelo de turbulencia LES: oneEqEddy, se predijo de manera más consistente el avance del flujo, donde otros métodos generaron salidas que se alejaban del comportamiento real del fluido en estudio. En el modelo físico se obtuvieron velocidades medias de $2.5784 \mathrm{~m} / \mathrm{s}$ y $2.1076 \mathrm{~m} / \mathrm{s}$, y coeficientes de determinación $R^{2}$ de 0.997 y 0.9903 respectivamente, y en el modelo numérico se obtuvo una velocidad media de $2.0572 \mathrm{~m} / \mathrm{s}$ y un $R^{2}$ de 0.974 , siendo las pendientes estadísticamente similares, brindando el modelo numérico un buen nivel de confiabilidad para poder ser utilizado para simular diferentes comportamientos de flujos de escombros en quebradas a escala natural con predicciones de manera adecuada. Asimismo, el modelo tridimensional de flujos de escombros reprodujo las velocidades del frente de onda con un $85 \%$ de precisión, coincidiendo en forma y tiempo en cada sección de control del modelo físico; los tirantes con un $80 \%$ de precisión y el alcance y desplazamiento del flujo de escombros dentro del cono de deyección. Los resultados del modelo experimental y de las simulaciones establecen que es posible modelar flujos de escombros en terrenos complejos con buena precisión.

Palabras clave- Flujo de escombros, software OpenFOAM, simulación numérica, quebrada experimental.
\end{abstract}

Abstract-OpenFOAM free software uses computational fluid dynamics through finite volume method; in adition, works the debris flow model with $98 \%$ precision in mesh refinement tests, applying the LES turbulence model: oneEqEddy. Likewise, the advance of the flow was predicted in a more consistent way, getting a behavior closer to the real one of the fluid under study comparing with other methods. In experimental model, average speeds between $2.5784 \mathrm{~m} / \mathrm{s}$ and $2.1076 \mathrm{~m} / \mathrm{s}$ were obtained, and a range $R^{2}$ determination coefficients of 0.997 to 0.9903; and in the numerical model an average speed of $2.0572 \mathrm{~m} / \mathrm{s}$ to $R^{2}$ of 0.974 were obtained,

Digital Object Identifier (DOI):

http://dx.doi.org/10.18687/LACCEI2020.1.1.570

ISBN: 978-958-52071-4-1 ISSN: 2414-6390 being the statistically similar slopes, providing the numerical model a good level of reliability to be used to simulate different behaviors of debris movement in ravines on a natural scale with accurate predictions. The three-dimensional model of debris flows reproduced the wavefront velocities with $85 \%$ precision, coinciding in shape and time in each control section of the physical model; Similarly, turnbuckles have gotten $80 \%$ accuracy, reach, and displacement of the debris flow within the ejection cone. The results of the experimental model and of the variable simulations stablish possible to model the debris flows in complex terrains with good precision.

Keywords- Debris flow, OpenFOAM software, numerical simulation, experimental ravines.

\section{INTRODUCCIÓN}

A lo largo de los años a nivel mundial se han realizado investigaciones importantes relacionadas al modelamiento de flujos de escombros. Se realizaron intentos iniciales en un programa con FORTRAN-77 para entradas de agua estables e inestables en canales, y para flujos de escombros con Reynolds menores a quinientos [1]; posteriormente, se hicieron modificaciones para convertir las ecuaciones de Manning a las ecuaciones de Chezy para modelar flujos de escombros laminares con incrementos de factores de fricción [2], luego se desarrollaron modelos de elementos finitos de dos y tres dimensiones para flujos de lodo aplicado a topografías superficiales simplificadas, usando el modelo reológico de Bingham cuyo esfuerzo al corte es una función de la viscosidad y el esfuerzo de cedencia del fluido [3]; más tarde, se modificaron tales modelos de flujos de escombros para incluir turbulencia [4].

Después se realizaron simulaciones integradas que permitían evaluar los cambios de las propiedades de los flujos de escombros debido a la erosión y deposición [5].

Finalmente, se adaptó un solver en OpenFOAM que permite modelar flujos de escombros en tres dimensiones y su impacto contra objetos, pudiendo simular diferentes escenarios cuyos parámetros pueden ser estimados según la composición del material [6].

18th LACCEI International Multi-Conference for Engineering, Education, and Technology: "Engineering, Integration, and Alliances for a Sustainable Development" "Hemispheric Cooperation for Competitiveness and Prosperity on a Knowledge-Based Economy”, July 27-31, 2020, Virtual Edition. 
En el Perú, se empleó el programa FLO-2D para simular flujos de escombros, concluyendo que es muy bueno; pero, con el fin de modelar flujos de lodos, cuyo material característico es un material fino, para simular flujo granular con una gran cantidad de material grueso es conveniente introducir datos de cada tipo de material, situación que el modelo no puede realizar [7]; así, también, usando el modelo numérico Kanako 2D se concluyó que este puede representar de manera adecuada los flujos de escombros, cuantificando la erosión y deposición de sedimentos, aunque es complicado validar los resultados al considerar homogeneidad geotécnica; del mismo modo, incluso, los caudales de flujos de escombros no son sensibles en magnitud a las variables geotécnicas, pues estos presentan la misma variabilidad que los hidrogramas líquidos, haciendo a la hidrología una variable vital para el tratamiento de flujos de escombros [8].

\section{A. Conceptos principales}

El SOF es un software libre de código abierto para la simulación de dinámica de fluidos computacional (DFC) desarrollado por OpenFOAM Foundation. Dicha biblioteca $\mathrm{C}++$ es usada principalmente para crear ejecutables, conocidos como aplicaciones, a su vez cuenta con una serie extensa de herramientas para el mallado, además del pre y posprocesamiento para la simulación de fluidos que son muy empleadas, tanto en las áreas de ingeniería cuanto en la investigación científica [9].

Large Eddy Simulation (LES) es un modelo matemático para la turbulencia utilizado en la dinámica de fluidos computacional que permite la simulación de grandes vórtices, con la idea principal de reducir el costo computacional ignorando las escalas de longitud más pequeñas [10].

El modelar el comportamiento tan complejo del flujo de escombros requiere de un solucionador capaz de diferenciar y caracterizar sus distintos componentes (flujo multifásico).

Este nuevo solucionador llamado debrisInterMixing Foam es una adaptación de interMixingFoam, uno de los solucionadores propios del SOF, el cual hace uso de la técnica numérica Volume Of Fluid (VOF) para trabajar la interface entre la mezcla de escombros y el aire, teniendo como datos de entrada, la topografía de la zona, las características geológicogeotécnicas del suelo, volumen de escombros, entre otros.

\section{B. Ecuaciones}

B.1. Ecuaciones de gobierno

El movimiento de la masa de escombros está definido por la ecuación de continuidad junto con las ecuaciones de transporte y momento, respectivamente.

$$
\begin{aligned}
& \nabla \cdot U=0 \\
& \frac{\partial \alpha_{m}}{\partial t}+\nabla \cdot\left(U \alpha_{m}\right)=0 \\
& \frac{\partial(\rho U)}{\partial t}+\nabla \cdot(\rho U U)=-\nabla p+\nabla \cdot T+\rho f
\end{aligned}
$$

Donde $U$ representa el campo de velocidad de la mezcla de escombros; $\alpha_{\mathrm{m}}$, la fracción de mezcla de fluido; $t$, el tiempo; $\rho$, la densidad de la fase promedio de la mezcla; $T$, la desviación del tensor de esfuerzo viscoso para la mezcla; $p$, la presión y $f$, las fuerzas de cuerpo por unidad de masa.

\section{B.2. Modelo reológico de Herschel Bulkley para sedimentos finos en suspensión \\ La reología de mezclas de agua con arcilla y arena es descrita por la ley reológica de Herschel - Bulkley [11].}

$\mu_{2}=k|\dot{\gamma}|^{n-1}+\tau_{y}|\dot{\gamma}|^{-1}$

Siendo $\mu_{2}$ la viscosidad dinámica del lodo, $\dot{\gamma}$ la tasa de corte, $\tau_{y}$ el esfuerzo de cedencia y $n$ define el comportamiento de adelgazamiento por corte $(\mathrm{n}<1)$ o espesamiento por corte $(\mathrm{n}>1)$.

\section{B.3. Modelo reológico viscoplástico de Coulomb para} representación de la grava

La representación de la grava mediante la reología viscoplástica de Coulomb dependiente de la presión y la tasa de deformación, se vuelve importante tan pronto como el material experimenta grandes presiones [12].

$\mu_{3}=\min \left(\mu_{0}, \mu_{\min }+\frac{p \cdot \sin (\delta)}{\frac{|| D||}{\frac{\rho}{\rho_{\text {mezcla }}}}}\left[1-e^{-m_{y}|| D||}\right]\right)$

Siendo $\mu_{3}$ la viscosidad dinámica de la grava; $\mu_{\min }$, la viscosidad dinámica mínima; $p$, la presión; $\delta$, el ángulo de fricción interno; $\|\mathrm{D}\|$, la norma del tensor de velocidad de deformación media y $\mathrm{m}_{\mathrm{y}}$, un parámetro numérico constante.

\section{METODOLOGÍA}

\section{A. Zona de estudio}

La quebrada experimental se encuentra ubicada en la quebrada Quilca, al noreste de la región Lima Metropolitana, provincia de Canta, distrito de Huamantanga (11 $229^{\prime} 56^{\prime} \mathrm{S}$, $\left.76^{\circ} 44^{\prime} 57^{\prime} \mathrm{O}\right)$, con una altura media de 3400 m.s.n.m.

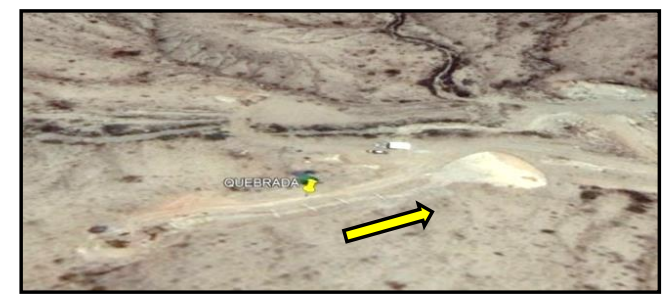

Fig. 1 Área de investigación experimental.

Hidrológicamente, la quebrada pertenece a la cuenca del río Chillón, el área experimental se muestra en la Fig. 1, la flecha señala el curso del agua.

$1^{\text {th }}$ LACCEI International Multi-Conference for Engineering, Education, and Technology: "Engineering, Integration, And Alliances for A Sustainable Development" "Hemispheric Cooperation for Competitiveness and Prosperity on a Knowledge-Based Economy", 29-31 July 2020, Buenos Aires, Argentina. 


\section{A.1. Descripción de la quebrada}

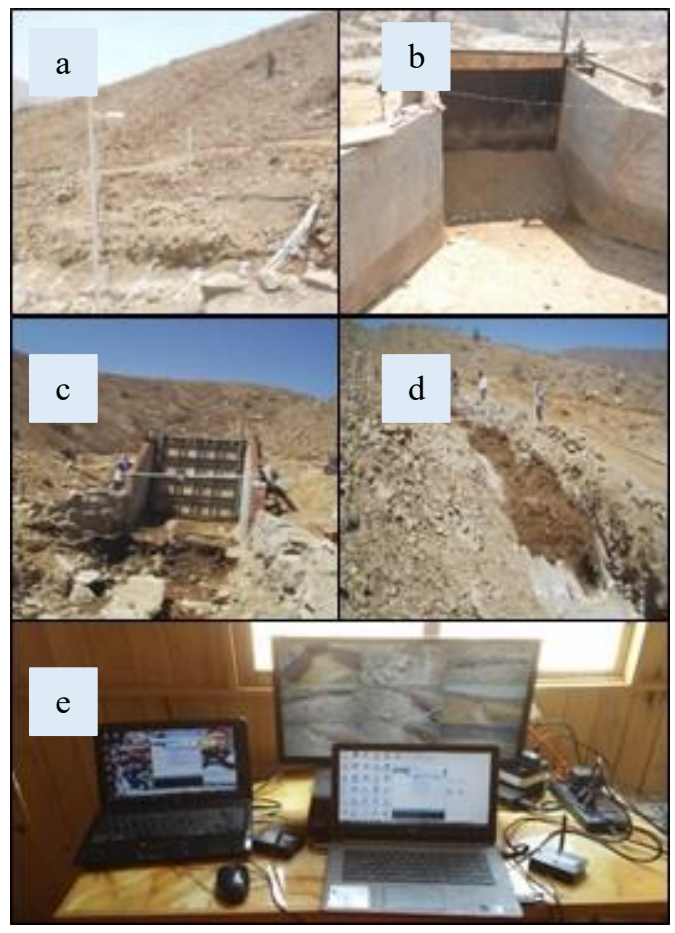

Fig. 2 Representación de las etapas del proceso: a) Instalación de equipos, b) Conformación del material c) Compuerta experimental, d) Avance del flujo de escombros, e) Monitoreo de datos.

La zona de la quebrada experimental se adaptó y posteriormente se instaló la instrumentación para la realización y medición de datos, ver Fig. 2.

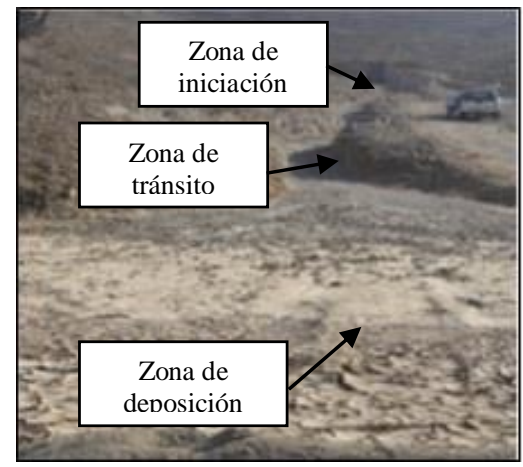

Fig. 3 Descripción de la quebrada experimental.

En la parte superior se encuentra el reservorio de material $(4,26 \mathrm{~m})$ y una compuerta de apertura manual (zona de iniciación), luego existe un tramo de $60 \mathrm{~m}$ con pendiente longitudinal promedio de $17 \%$ y un cauce de aproximadamente $2 \mathrm{~m}$ de ancho, denominado la zona de tránsito del flujo de escombros; finalmente, en la parte inferior se encuentra el cono de deyección o zona de deposición de aproximadamente $20 \mathrm{x}$ 20 metros, ver Fig. 3.

\section{A.2. Caracterización geológico-geotécnica, caracterización físico-análisis granulométrico-mineralogía}

El material sólido usado para la conformación del volumen de escombros inicial fue clasificado como SM Arena limosa con grava y estuvo compuesto de $23,7 \%$ de gravas, 63,3 $\%$ de arena y $12,9 \%$ de finos $(6,8 \%$ limos y $6.1 \%$ arcilla).

La gravedad específica del material fue de 2.89 y el peso unitario de $1709 \mathrm{~kg} / \mathrm{m}^{3}$. Asimismo, la composición mineralógica fue de $50 \%$; Plagioclasa, $16 \%$; Cuarzo, $11 \%$; Feldespato-K, $8 \%$; Anfíbol, $5 \%$; mineral laminar, $4 \%$; Mica y $3 \%$, Clorita.

\section{B. Metodología experimental}

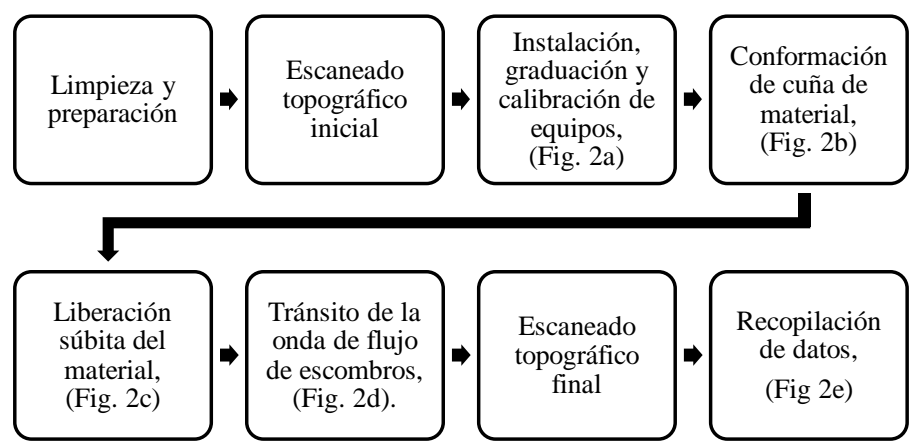

Fig. 4 Diagrama de flujo de la metodología experimental.

TABLA I

SECCIONES DE CONTROL

\begin{tabular}{|c|c|}
\hline $\begin{array}{c}\text { SECCIÓN DE } \\
\text { CONTROL }\end{array}$ & $\begin{array}{c}\text { PROGRESIVA } \\
(\mathrm{m})\end{array}$ \\
\hline 01 & $0+002$ \\
\hline 02 & $0+008$ \\
\hline 03 & $0+020$ \\
\hline 04 & $0+027$ \\
\hline 05 & $0+035$ \\
\hline 06 & $0+043$ \\
\hline 07 & $0+052$ \\
\hline
\end{tabular}

Fuente: Datos recopilados del modelo experimental [13].

La Fig. 4, resume todo el desarrollo de un ensayo experimental de flujo de escombros, desde antes de iniciado el evento hasta la toma posterior de datos experimentales, monitoreados en siete secciones de control, ver Tabla I.

\section{Metodología para la simulación numérica \\ C.1. Generación de la Malla}

Haciendo uso del SOF para la simulación, el primer paso fue crear una malla de fondo estructurada usando el generador de mallas blockMesh del SOF, que cubra la superficie total de la quebrada donde se definieron primero los vértices.

$1^{\text {th }}$ LACCEI International Multi-Conference for Engineering, Education, and Technology: "Engineering, Integration, And Alliances for A Sustainable Development" "Hemispheric Cooperation for Competitiveness and Prosperity on a Knowledge-Based Economy”, 29-31 July 2020, Buenos Aires, Argentina. 


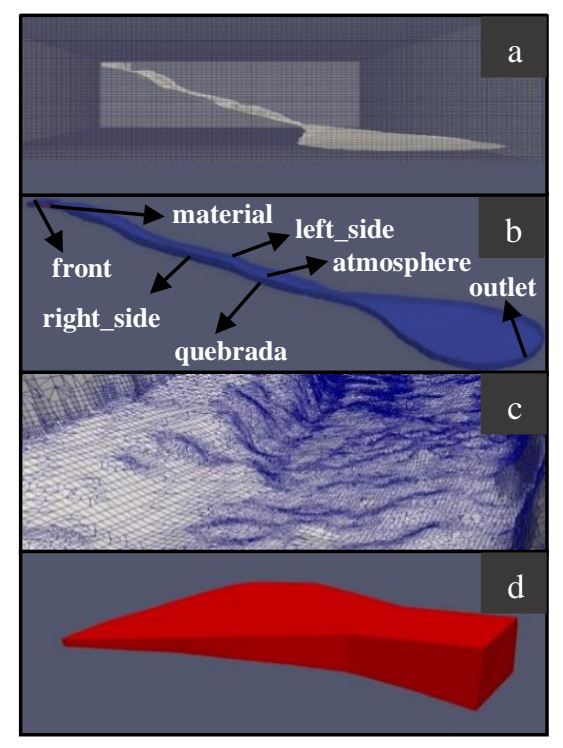

Fig. 5 Representación: a) Superficie de la quebrada dentro de la malla de fondo estructurada, b) Malla final de la quebrada, c) Tramo interior de la malla final, d) Superficie del material.

Posteriormente, el bloque de hexaedros, indicando el número de celdas en cada dirección y la relación de expansión de las mismas.

Superficie de la quebrada (Fig. 5a).

- Límites (x y z):

Mínimo y máximo:

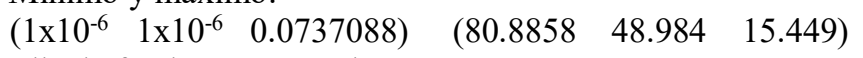

Malla de fondo estructurada:

- Límites (x y z):

Mínimo y máximo:

$\left(\begin{array}{lllll}-1.000-1.000-1.000) & (81.000 & 49.000 & 16.000\end{array}\right)$

- Vértices del bloque: (0 122345567 )

- Número de celdas en x, y, z: (820 500 170)

- Relación de expansión de las celdas: $\left(\begin{array}{lll}1 & 1 & 1\end{array}\right)$

Finalmente, se usó el generador de mallas tridimensionales snappyHexMesh para realizar el mallado debido a la complejidad de la superficie, donde se indicaron los parches de contorno (Fig. 5b), así como parámetros para el refinamiento de la malla, (Fig. 5c).

TABLA II

CARACTERÍSTICAS DE LA MALLA FINAL

\begin{tabular}{|c|c|c|c|}
\hline \multirow{2}{*}{$\mathrm{N}^{\circ}$ de Puntos } & \multirow{2}{*}{$\mathrm{N}^{\circ}$ de Celdas } & $\begin{array}{c}|c| \\
\mathrm{N}^{\circ} \text { de caras } \\
\text { internas }\end{array}$ & $\begin{array}{c}\mathrm{N}^{\circ} \text { de caras de } \\
\text { contorno }\end{array}$ \\
\hline 3386395 & 2849972 & 8474865 & 524667 \\
\hline
\end{tabular}

Así, también, se crea una superficie que representa el volumen del material del flujo de escombros (lodo + grava) dentro del reservorio (H: 1,0 m - Cv: $40 \%$ ). (315488 celdas), (Fig. 5d). Las características de la malla final se muestran en la Tabla II.

\section{C.2. Propiedades del Fluido}

TABLA III

PARÁMETROS DEL MODELO REOLÓGICO HERSCHEL BULKLEY

\begin{tabular}{|c|c|c|c|}
\hline Parámetro & Descripción & Valor & Unidad \\
\hline Contenido de agua & - & 0.6 & - \\
\hline $\mathrm{C}_{\mathrm{v}}$ & $\begin{array}{l}\text { Concentración } \\
\text { volumétrica de sólidos }\end{array}$ & 0.18796 & - \\
\hline $\mathrm{P}_{0 \mathrm{v}}$ & $\begin{array}{l}\text { Porcentaje volumétrico } \\
\text { de arcilla }\end{array}$ & 0.06576 & - \\
\hline$\tau_{00}$ & $\begin{array}{l}\text { Parámetro libre calibrado } \\
\text { según ensayos de } \\
\text { laboratorio. }\end{array}$ & 30 & $\mathrm{~Pa}$ \\
\hline$\rho_{\mathrm{F}}$ & $\begin{array}{l}\text { Densidad aparente de la } \\
\text { mezcla }\end{array}$ & 1358 & $\mathrm{~kg} / \mathrm{m}^{3}$ \\
\hline$v_{0}$ & $\begin{array}{l}\text { Viscosidad cinemática } \\
\text { máxima }\end{array}$ & 2 & $\mathrm{~m}^{2} / \mathrm{s}$ \\
\hline$\tau_{0}$ & $\begin{array}{l}\text { Esfuerzo modificado } \\
\text { según la concentración } \\
\text { volumétrica de sólidos. }\end{array}$ & 0.02209 & $\mathrm{~m}^{2} / \mathrm{s}^{2}$ \\
\hline $\mathrm{k}$ & Índice de consistencia & 0.00031 & $\mathrm{~m}^{2} / \mathrm{s}$ \\
\hline $\mathrm{n}$ & $\begin{array}{l}\text { Índice } \\
\text { comportamiento } \\
\text { (calibrado según modelo } \\
\text { experimental) }\end{array}$ & 0.34 & - \\
\hline
\end{tabular}

Para el lodo (mezcla de agua + sedimentos finos), según el modelo reológico de Herschel Bulkley, en la Tabla III se muestran los parámetros requeridos [13].

TABLA IV

PARÁMETROS DEL MODELO REOLÓGICO VISCOPLÁSTICO DE COULOMB

\begin{tabular}{|c|l|c|c|}
\hline Parámetro & \multicolumn{1}{|c|}{ Descripción } & Valor & Unidad \\
\hline$\delta$ & $\begin{array}{l}\text { Ángulo de fricción interna de la } \\
\text { grava }\end{array}$ & $\begin{array}{c}37^{\circ} \\
(0.645772)\end{array}$ & $\mathrm{rad}$ \\
\hline$\rho_{\mathrm{S}}$ & Densidad de la mezcla & 1358 & $\mathrm{~kg} / \mathrm{m}^{3}$ \\
\hline$v_{\mathrm{min}}$ & Viscosidad cinemática mínima & $1 \times 10^{-6}$ & $\mathrm{~m}^{2} / \mathrm{s}$ \\
\hline$v_{0}$ & Viscosidad cinemática máxima & 2 & $\mathrm{~m}^{2} / \mathrm{s}$ \\
\hline $\mathrm{m}_{\mathrm{y}}$ & $\begin{array}{l}\text { Parámetro numérico calibrado } \\
\text { según modelo experimental }\end{array}$ & 0.2 & $\mathrm{~s}$ \\
\hline
\end{tabular}

Así mismo, para la grava, la Tabla IV nos presenta el modelo reológico Viscoplástico de Coulomb [13].

TABLA V

PARÁMETROS ADICIONALES

\begin{tabular}{|c|l|c|c|}
\hline Parámetro & \multicolumn{1}{|c|}{ Descripción } & Valor & Unidad \\
\hline$\sigma_{12}$ & $\begin{array}{l}\text { Tensión superficial (aire - } \\
\text { lodo) }\end{array}$ & 0.0762 & $\mathrm{~kg} / \mathrm{s}^{2}$ \\
\hline$\sigma_{13}$ & $\begin{array}{l}\text { Tensión superficial (aire - } \\
\text { grava) }\end{array}$ & 0.0762 & $\mathrm{~kg} / \mathrm{s}^{2}$ \\
\hline$D_{23}$ & $\begin{array}{l}\text { Difusividad entre el lodo y } \\
\text { la grava }\end{array}$ & 0 & $\mathrm{~m}^{2} / \mathrm{s}$ \\
\hline$\rho_{\mathrm{S}}$ & Densidad de la grava & 2700 & $\mathrm{~kg} / \mathrm{m}^{3}$ \\
\hline$\varnothing_{\mathrm{S}}$ & Diámetro de la grava & 0.019085 & $\mathrm{~m}$ \\
\hline
\end{tabular}

$18^{\text {th }}$ LACCEI International Multi-Conference for Engineering, Education, and Technology: "Engineering, Integration, And Alliances for A Sustainable Development" "Hemispheric Cooperation for Competitiveness and Prosperity on a Knowledge-Based Economy”, 29-31 July 2020, Buenos Aires, Argentina. 
En la Tabla V se expresan parámetros adicionales, incluyendo la aceleración de la gravedad un valor de $9.81 \mathrm{~m} / \mathrm{s}^{2}$ [13].

\section{C.3. Modelo de Turbulencia}

Se realizaron distintas pruebas con modelos de turbulencia RAS y LES, donde el modelo de turbulencia de mejores resultados y el que más se ajusta al modelo experimental de flujos de escombros fue: LES: oneEqEddy para flujos incompresibles. Hay que considerar también, que según la concentración de sedimentos y/o parámetros del evento experimental, el modelo de turbulencia puede variar debido a las distintas condiciones propias de un evento real.

\section{RESULTADOS}

\section{A. Escenario de resultados y calibración}

Se consideraron los parámetros de los ensayos Q-H1-C40; es decir, con un tirante inicial de $1.00 \mathrm{~m}$ y concentración de sedimentos de $40 \%$, por ser los más críticos.

\section{B. Posición del frente de onda vs tiempo}

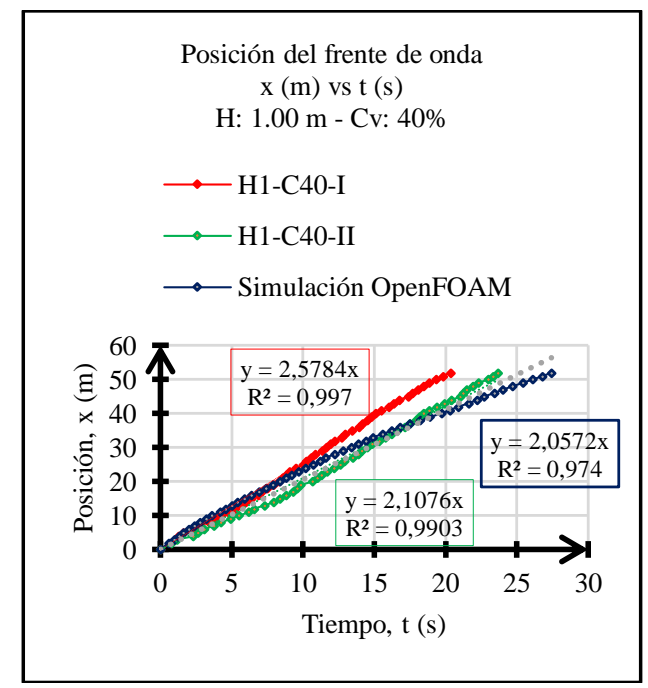

Fig. 6 Posición del frente de onda del flujo de escombros para $\mathrm{H}: 1,00 \mathrm{~m}$ y Cv: $40 \%$.

Según la Fig. 6, el avance del frente de onda del flujo de escombros en el modelo experimental tiene una tendencia lineal, para el primer ensayo se tiene una velocidad media de $2.5784 \mathrm{~m} / \mathrm{s}$ y para el segundo, $2.1076 \mathrm{~m} / \mathrm{s}$. Así también se considera tal metodología como válida ya que para el primer ensayo se tiene un coeficiente de determinación $\mathrm{R}^{2}=0.997 \mathrm{y}$ para el segundo, un $\mathrm{R}^{2}=0.9903$, es decir que el modelo numérico tiene un buen ajuste con las variables reales de posición y velocidad. Del mismo modo para el modelo simulado en OpenFOAM, se tiene una velocidad media de $2.0572 \mathrm{~m} / \mathrm{s}$ y un $\mathrm{R}^{2}=0.974$, equivalente a $97.4 \%$, considerando que la metodología utilizada en el modelo es adecuada.
De la misma manera, se muestra la comparación del evento real (a) y con el evento simulado (b) según el tránsito del flujo de escombros en tres secciones de control significativas.

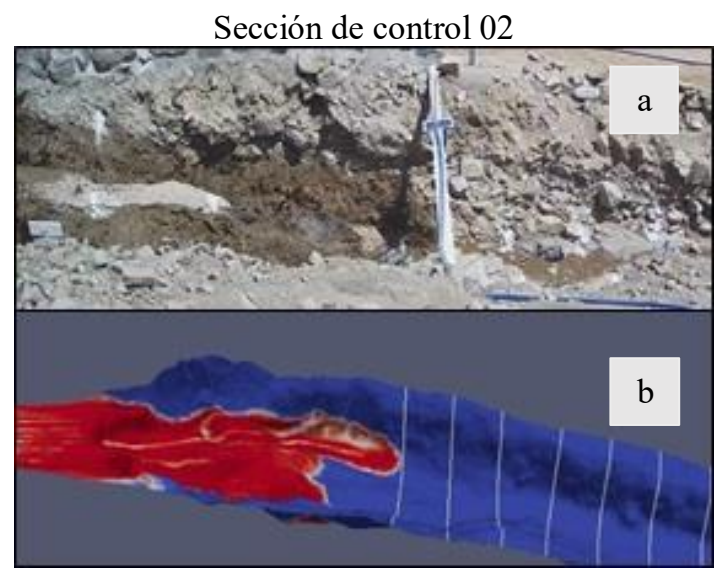

Fig. 7 Comparación de los modelos (a) y (b) en la sección 02.

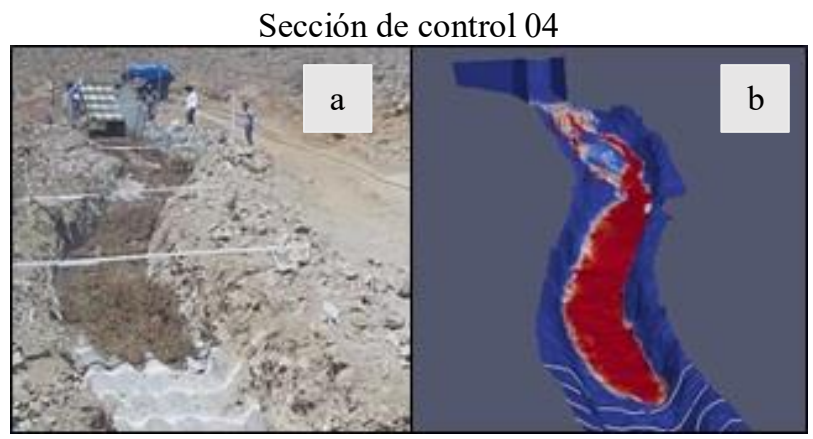

Fig. 8 Comparación de los modelos (a) y (b) en la sección 04.

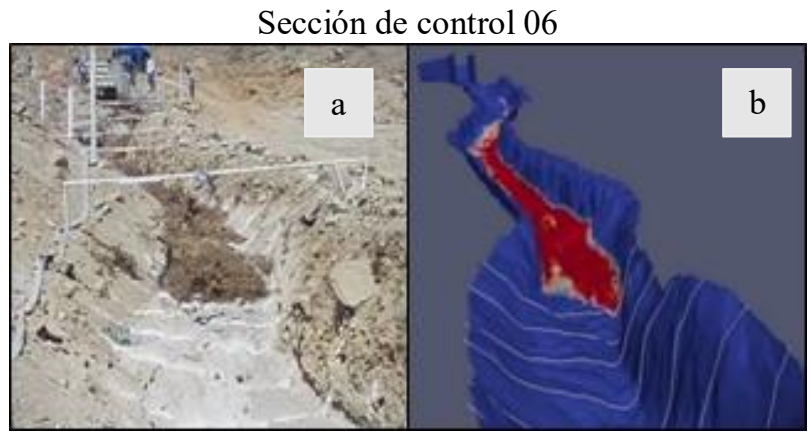

Fig. 9 Comparación de los modelos (a) y (b) en la sección 06.

Según (Fig. 7, 8 y 9), puede apreciarse que el frente de onda del evento experimental tiene características similares al modelo simulado en OpenFOAM, pues coinciden en la misma zona del cauce de la quebrada para un mismo tiempo.

$18^{\text {th }}$ LACCEI International Multi-Conference for Engineering, Education, and Technology: "Engineering, Integration, And Alliances for A Sustainable Development" "Hemispheric Cooperation for Competitiveness and Prosperity on a Knowledge-Based Economy”, 29-31 July 2020, Buenos Aires, Argentina. 


\section{Evento finalizado de flujo de escombros}

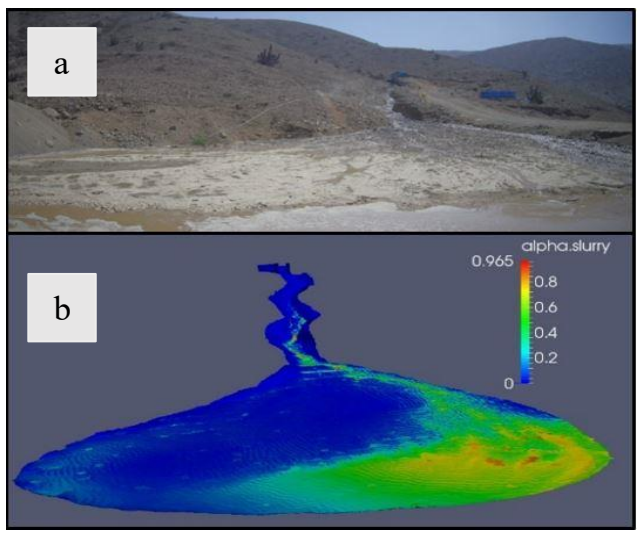

Fig. 10 Comparación de modelos (a) y (b) para t $=100$ s.

Se puede apreciar que el flujo de escombros tiende a sedimentar por la margen derecha del cauce, mientras que se produce mayor erosión por la margen izquierda, tanto en el modelo físico cuanto en el numérico, (Fig. 10).

D. Análisis de erosión - sedimentación

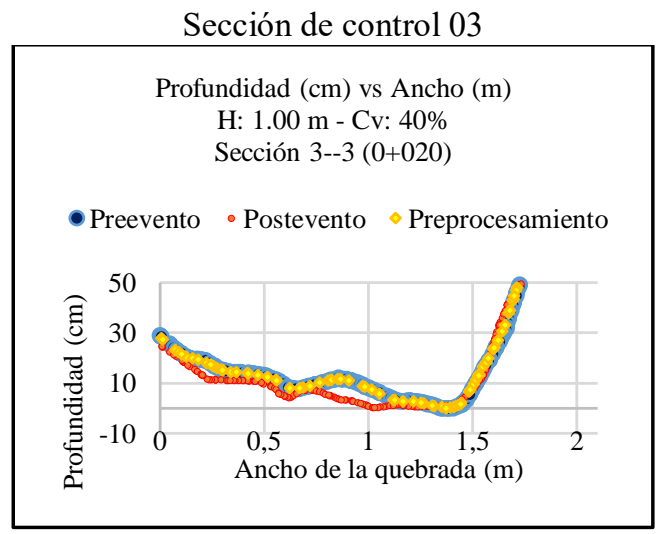

Fig. 11 Área de sección transversal 03, quebrada experimental.

Sección de control 04

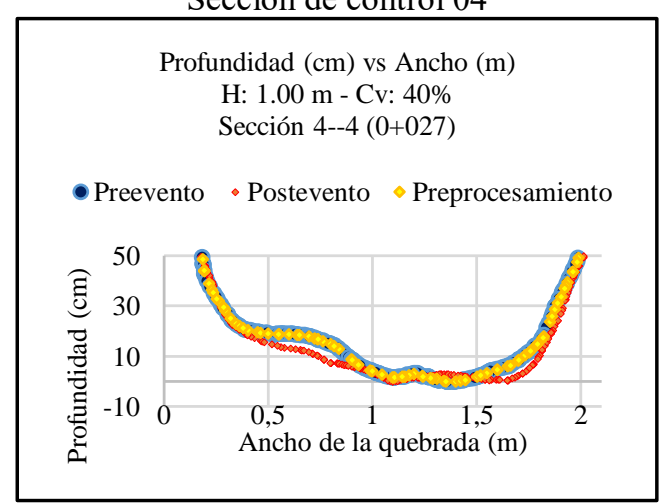

Fig. 12 Área de sección transversal 04, quebrada experimental.
Sección de control 05

Profundidad (cm) vs Ancho (m)

H: $1.00 \mathrm{~m}-\mathrm{Cv}: 40 \%$

Sección 5--5 (0+035)

- Preevento Postevento Preprocesamiento

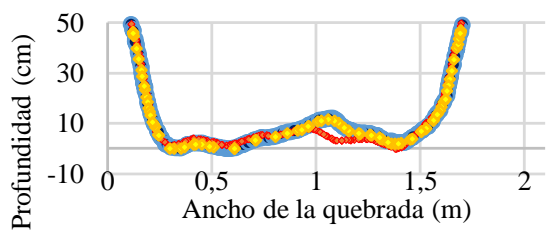

Fig. 13 Área de sección transversal 05, quebrada experimental.

Haciendo el análisis de erosión-sedimentación, se verifica que el flujo de escombros tiende a sedimentar por la margen derecha del cauce, mientras que se produce mayor erosión por la margen izquierda de la misma, (Fig. 11, 12 y 13).

Otro parámetro de evaluación fue el tirante de flujo, para ello se evaluó en tres secciones de control significativas; la calibración consistió en coincidir los tirantes del modelo físico con los tirantes del modelo numérico.

\section{E. Tirante vs Tiempo}

\section{Sección de control 02}

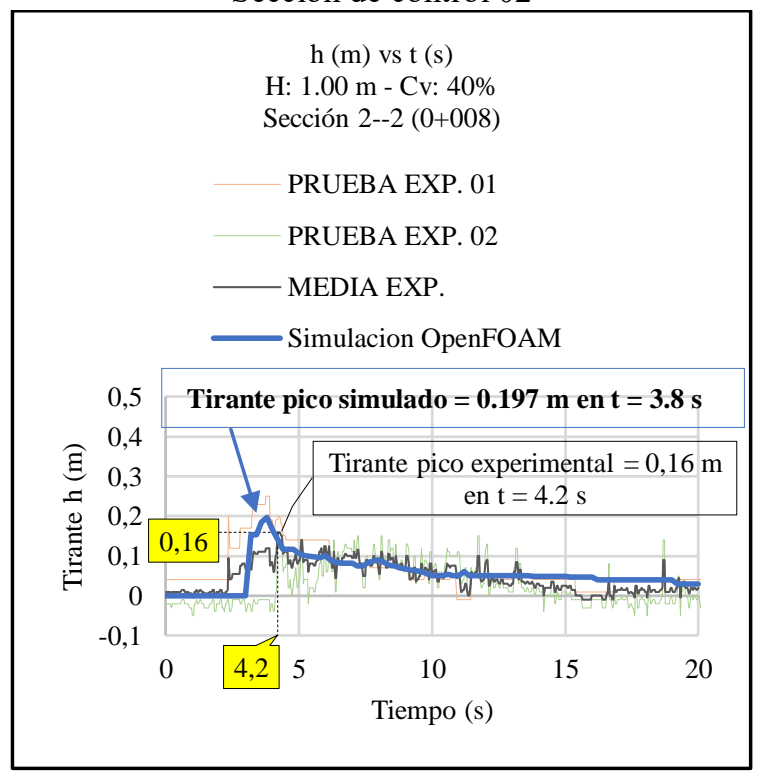

Fig. 14 Hidrograma del tirante del flujo de escombros vs. tiempo en la sección de control $02(0+008)$.

$1^{\text {th }}$ LACCEI International Multi-Conference for Engineering, Education, and Technology: "Engineering, Integration, And Alliances for A Sustainable Development" "Hemispheric Cooperation for Competitiveness and Prosperity on a Knowledge-Based Economy”, 29-31 July 2020, Buenos Aires, Argentina. 
Sección de control 05

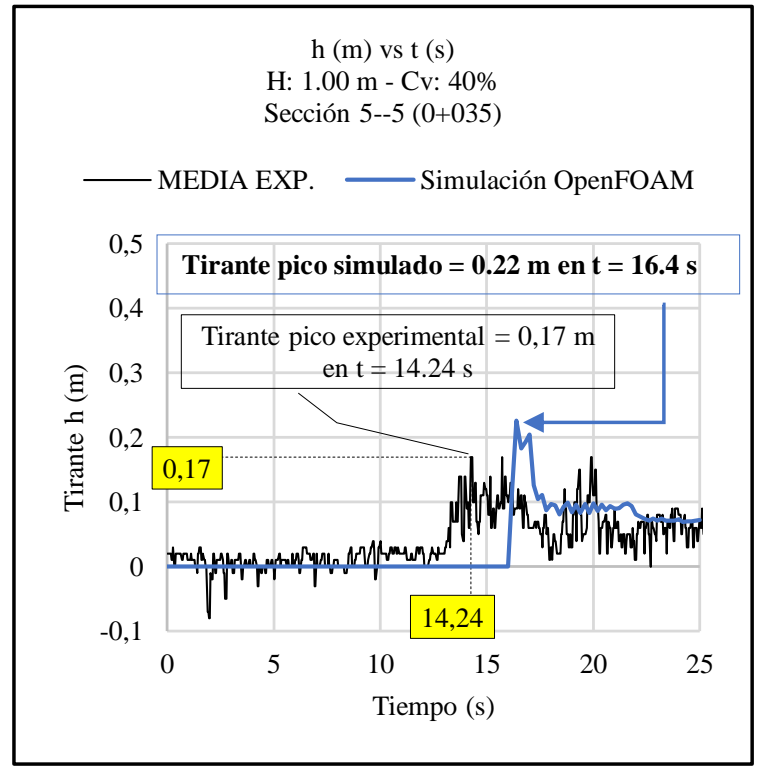

Fig. 15 Hidrograma del tirante del flujo de escombros vs. tiempo en la sección de control $05(0+035)$.

Sección de control 07

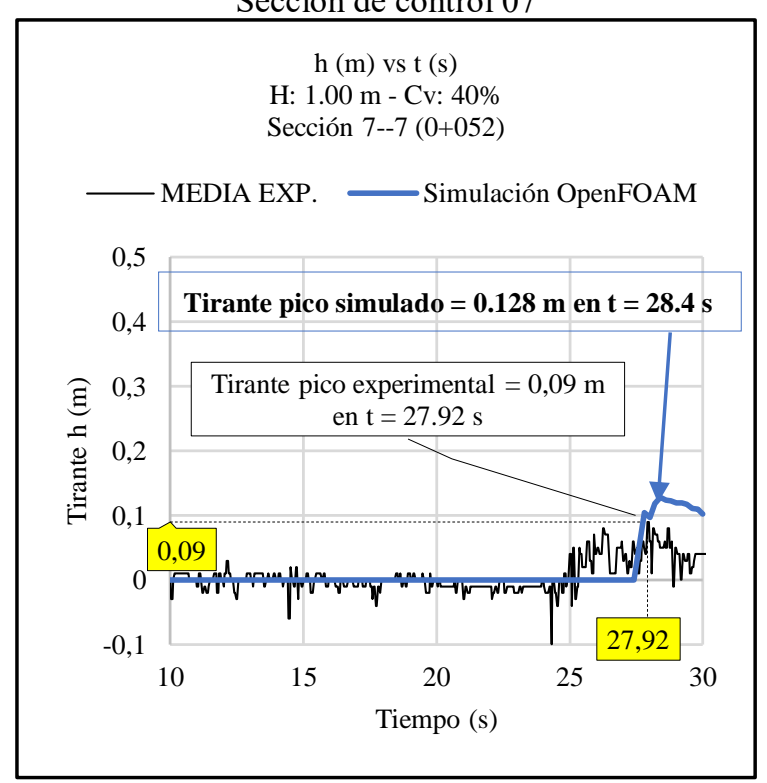

Fig. 16 Hidrograma del tirante del flujo de escombros vs. tiempo en la sección de control $07(0+052)$.

Debido a que se asumen condiciones iniciales a un nivel macroscópico, la dispersión obtenida (Fig. 14, 15 y 16) revela los efectos de detalles incontrolables, tales como errores de alineación, medición, condiciones ambientales, tolerancias propias del sensor, etc. Sin embargo, permiten reproducir las características principales de los flujos de escombros, pudiendo ser usados sin ningún problema para tales fines.

\section{DISCUSIÓN}

Muchas de las investigaciones realizadas atribuyen que los flujos de escombros fluyen con una reología fija, no newtoniana. [2] asumiendo que los flujos de escombros son laminares. Por lo tanto, desarrollaron ensayos experimentales incrementando los factores de fricción. Sin embargo, en esta investigación quedó en evidencia que, en la mayoría de los casos, los flujos de escombros típicos tienen un comportamiento turbulento y el mejor ajuste se produjo con la metodología LES.

En trabajos similares se realizaron simulaciones integradas para evaluar los cambios de las propiedades de los flujos de escombros debido a la erosión y deposición. En este modelo numérico, no se evaluó la erosión del lecho, debido a que se requiere aplicar deformación de malla, lo cual está fuera del alcance de esta investigación; no obstante, esta fue realizada con un $98 \%$ de precisión [5].

En otros estudios se adaptaron un solver para modelar flujos de escombros en tres dimensiones y su impacto contra objetos en escenarios cuyos parámetros pueden estimarse según la composición del material; empero, trabajaron con geometrías simples [6]. Por el contrario, en esta investigación se usó una quebrada cuasirreal cuyo mallado se realizó con las herramientas propias del SOF, así como la inclusión de turbulencia, propia de los flujos de escombros típicos, pudiendo usar tal metodología para simular distintos tipos de eventos.

Usando el modelo bidimensional FLO-2D se concluyó que es muy bueno, pero para modelar flujos de lodos, cuyo material característico es un material fino; puesto que, para simular flujo granular con una gran cantidad de material grueso es conveniente introducir los datos de cada tipo de material, porque el modelo no puede realizar [7]; caso contrario sucede con el modelo OpenFOAM desarrollado en esta investigación, el cual puede modelar flujos multifásicos, haciendo distinción entre los distintos componentes de un flujo de escombros típico.

\section{CONCLUSIONES}

El comportamiento del flujo, reflejado en la posición del frente de onda y otras variables, varía según el nivel de concentración de sedimentos, siendo conscientes que los modelos reológicos usados aún son limitados.

Para adaptar el modelo de flujos de escombros, se realizaron pruebas correspondientes de refinamiento de malla, logrando un $98 \%$ de precisión; asimismo, el algoritmo que predijo de manera más precisa el avance del flujo fue LES: oneEqEddy. Otros métodos generaron salidas que se alejaban del comportamiento real del flujo en la quebrada estudiada.

Para el modelo físico se obtuvieron velocidades medias de $2.5784 \mathrm{~m} / \mathrm{s}$ y $2.1076 \mathrm{~m} / \mathrm{s}$, y coeficientes de determinación $\mathrm{R}^{2} \mathrm{de}$ 0.997 y 0.9903 , respectivamente; es decir, el modelo representa de manera adecuada el comportamiento del flujo en la quebrada a escala natural. Para el modelo numérico, se obtuvo una velocidad media de $2.0572 \mathrm{~m} / \mathrm{s}$ y un $\mathrm{R}^{2}$ de 0.974 , siendo las pendientes estadísticamente similares, considerando así que la

$18^{\text {th }}$ LACCEI International Multi-Conference for Engineering, Education, and Technology: "Engineering, Integration, And Alliances for A Sustainable Development" "Hemispheric Cooperation for Competitiveness and Prosperity on a Knowledge-Based Economy", 29-31 July 2020, Buenos Aires, Argentina. 
metodología usada tiene un alto nivel de confiabilidad y puede ser aplicable para simular distintos tipos de eventos.

El modelo tridimensional de flujos de escombros reprodujo las velocidades del frente de onda con un $85 \%$ de precisión, coincidiendo en forma y tiempo en cada sección de control del modelo físico; los tirantes con un 80 \% de precisión y el alcance y desplazamiento del flujo de escombros dentro del cono de deyección.

\section{AGRADECIMIENTOS}

A la Universidad Nacional de Ingeniería y la Universidad Nacional del Santa. La ejecución de este proyecto formó parte del Convenio $\mathrm{N}^{\circ}$ 257-INNOVATEPERU-EC-2016, firmado entre la Universidad Nacional de Ingeniería y el Programa Nacional de Innovación para la Competitividad y Productividad del Ministerio de la Producción, INNOVATE-PERÚ,

Al Laboratorio Nacional de Hidráulica (UNI), en especial al Dr. Julio Kuroiwa Zevallos y al Ing. Luis Castro.

Al IMEFEN, en especial gratitud al Ing. Roger Hidalgo, por acoger y permitir trabajar con su institución durante el desarrollo del proyecto de investigación experimental de huaicos.

\section{REFERENCIAS}

[1] R. Jeppson, "Simulation of steady and unsteady flows in channels and rivers", Utah Water Research Laboratory, Utah State University, Logan, Utah, Reports. Paper, pp. 301, 1974.

[2] A. DeLeon, and R. Jeppson, "Hydraulics and Numerical Solutions of Steady-State but Spatially Varied Debris Flow", Reports. Paper, pp. 515, 1982.

[3] R. MacArthur, and D. Schamber, "Numerical methods for simulating mudflows", Proc. of the 3rd Intl. Symp. On River Sedimentation, Univ. of Mississipi, pp. 1615-1623, 1986.

[4] T. Takahashi, and H. Nakagawa, "Debris flow hazard zone mapping", Proc. of the Japan - China (Taipai) Joint Seminar on Natural Hazard Mitigation. Kyoto, Japan, pp. 363-372, 1989.

[5] H. Chen, and L. Zhang, "EDDA 1.0: integrated simulation of debris flow erosion, deposition and property changes", Geosci. Model Dev., 8. pp. 829-844, 2015.

[6] A. Boetticher, J. Turowski, B. McArdell, D. Rickenmann, M. Hürlimann, C. Scheidl, and J. Kirchner "DebrisInterMixing-2.3: a Finite Volume solver for three dimensional debris flow simulations based on a single calibration parameter", Geosci. Model Dev., 9. pp. 29092923, 2016.

[7] L. Castillo, "Aplicación de un modelo numérico de flujos de escombros y lodo en una quebrada en el Perú", Tesis de pregrado, Universidad Nacional de Ingeniería, Lima, Perú, pp. 173-179, 2006.
[8] F. Escusa, "Análisis y modelamiento de flujos de escombros en la zona de Maranura, La convención Cusco", Tesis de pregrado, Pontificia Universidad Católica del Perú, Lima, Perú, pp. 96-97, 2016.

[9] The OpenFOAM Foundation, "OpenFOAM v6 User Guide", London, UK: CFD Direct Ltd, 2018 [Recuperado de https://cfd.direct/openfoam/user-guide].

[10] J. Bo, "Implementation and assessment of subgrid-scale models for large eddy simulations of incompressible turbulent flows", Tesis para el grado de Master en ciencias, Universidad de Oslo, Noruega, pp. 1-5, 2015.

[11] P. Coussot, D. Laigle, M. Aratano, A. Deganuttil, and L. Marchi, "Direct determination of rheological characteristics of debris flow", J. Hydraul. Eng. - ASCE, (124). pp. 865-868, 1998.

[12] B. Domnik, and S. Pudasaini, "Full two-dimensional rapid chute flows of simple viscoplastic granular materials with a pressure dependent dynamic slip-velocity and their numerical simulations", J. Non-Newton. Fluid. pp. 173174, 72-86, 2012.

[13] UNI-UNALM-SQ\&ICC, "Estudio Experimental de la Dinámica de Flujos de Huaycos a Escala Cuasi-Real en Ríos y Quebradas de Alta Pendiente", Proyecto de Investigación C-359-PNICP-PIAP-2014. Informe Técnico $N^{\circ}$ 3, IMEFEN-UNI, FIA-UNALM, SQ\&ICC, Lima, Perú, 2018.

18 $^{\text {th }}$ LACCEI International Multi-Conference for Engineering, Education, and Technology: "Engineering, Integration, And Alliances for A Sustainable Development" "Hemispheric Cooperation for Competitiveness and Prosperity on a Knowledge-Based Economy”, 29-31 July 2020, Buenos Aires, Argentina. 